\title{
Magnetic polaritons in metamagnet layered structures: Spectra and localization properties
}

\author{
C.A.A. Araújo ${ }^{\text {a }}$, E.L. Albuquerque ${ }^{\mathrm{a}, *}$, D.H.A.L. Anselmo ${ }^{\text {a }}$, M.S. Vasconcelos ${ }^{\mathrm{b}}$ \\ ${ }^{a}$ Departamento de Física, Universidade Federal do Rio Grande do Norte, 59072-970 Natal-RN, Brazil \\ ${ }^{\mathrm{b}}$ Departamento de Ciências Exatas, Centro Federal de Educação Tecnológica do Maranhão, 65025-001 São Luís-MA, Brazil
}

Received 18 July 2007; accepted 6 September 2007

Available online 11 September 2007

Communicated by R. Wu

\begin{abstract}
The magnetic polariton propagation in metamagnet layered structures is theoretically studied by using a transfer matrix approach. The layered structures considered here are made up by the stacking of two different layers (also known as building blocks, named $A$ and $B$ ), where one of them is a metamagnetic thin film $(A)$, while the other is a non-magnetic insulator thin layer $(B)$. We take into account both the antiferromagnetic $(\mathrm{AFM})$ and ferromagnetic (FM) phases of the metamagnetic material. For the periodic arrangement, the bulk modes are characterized by two large symmetric bands, with non-reciprocal surface modes between them. The quasiperiodic metamagnetic structure is then built up by following the Fibonacci sequence, whose long-range order effect is then investigated, giving rise to an interesting self-similar spectra and a power-law profile.
\end{abstract}

(C) 2007 Elsevier B.V. All rights reserved.

PACS: 71.36.+c; 74.70.-i; 75.70.Cn; 61.44.-n

Keywords: Magnetic polaritons; Layered structures; Metamagnetic materials; Self-similar spectra

\section{Introduction}

Since the discovery of the icosahedral phase on an Al-Mn alloy by Shechtman et al. [1], researches on the subject of quasiperiodic structures (QPS) have attracted a lot of attention. A fascinating feature of these structures is that they exhibit collective properties not shared by their constituent parts. Furthermore, the long-range correlations induced by the construction of these systems are expected to be reflected to some degree in their various spectra (as in light propagation, electronic transmission, density of states, polaritons, etc.), defining a novel description of disorder. Indeed, theoretical transfer matrix treatments show that these spectra are fractals (for an up to date review see Refs. [2,3]).

\footnotetext{
* Corresponding author. Tel.: +55 84 32153793; fax: +55 8432153791.

E-mail address: eudenilson@dfte.ufrn.br (E.L. Albuquerque).
}

Advances in the fabrication of multilayer structures and its characterization, such as neutron diffraction and X-ray scattering, provide the possibility to reveal their novel features, giving a fair physical background, which spans from methods based on numerical simulation to rigorous mathematical demonstrations [4]. Furthermore,they form a new class of intriguing materials, where their macroscopic properties are designed (or controlled) by varying the thickness (or composition) of the constituent films. In fact, some of these properties may be unique to the multilayer structures and provide the potential for device applications [5].

Although the spin waves propagation on anisotropic metamagnets $\mathrm{FeCl}_{2}$ and $\mathrm{FeBr}_{2}$ was already previously studied [6,7], and temperature effects on these spectra were also considered $[8,9]$, for the best of our knowledge nothing was published so far concerning the propagation, in metamagnet layered structures, of the mixed mode which arises from a magnon (the quantum of a spin wave)-photon interaction, the so-called mag- 
netic polaritons, which yields a much richer spectrum. It is therefore our aim to fill this gap by presenting in this work a comprehensive investigation of localization and scaling properties of the magnetic polaritons that can propagate in magnetic multilayer structures made up of materials $A$ (metamagnet) and $B$ (insulator) stacked alternately, by following the Fibonacci quasiperiodic mathematical sequence. Our model is based on a transfer matrix approach, in order to simplify the algebra, which is otherwise quite involved. The metamagnetic materials consist of ferromagnetically ordered layers, with the intralayer ferromagnetic exchange interactions being much stronger than the weak antiferromagnetic interaction between adjacent layers. We consider also the presence of a weak external magnetic field $\vec{H}$ applied perpendicular to the layers. In the regime of low temperatures and for small values of the external magnetic field $\vec{H}$, the adjacent layers of the metamagnet material order antiparallel to one another, giving the antiferromagnetic (AFM) phase. On the other hand, for larger $\vec{H}$ enough to overcome the interlayer antiferromagnetic coupling, the overall ordering is ferromagnetic (FM phase). Both cases will be considered in this work.

The plan of this Letter is as follows: in Section 2, we present the method of calculation employed here, which is based on the transfer matrix approach. The magnetic polariton dispersion relation (bulk and surface modes) is then determined. Section 3 is devoted to the discussion of the polariton's dispersion relation for the periodic and quasiperiodic structures. Further, we present also their localization profiles and the connection with a fractal/multifractal behavior through the scaling law of their bandwidth spectra.

\section{General theory}

Before to treat the general problem of the QPS, it is more intuitive to deal first with the simpler periodic case, in which the building blocks $A$ (metamagnet) and $B$ (insulator) are arranged in an alternating way $A B A B \cdots$. It is displayed in a geometry such that the $z$-axis coordinate is placed parallel to the easy-axis of the layers. The thickness of the magnetic (nonmagnetic) layer is represented by $d_{A}\left(d_{B}\right)$, and therefore the unit-cell thickness is given by $L=d_{A}+d_{B}$. It fills the semispace $z \leqslant 0$, with its surface parallel to the $x y$ plane. On the $z \geqslant 0$ region we have vacuum. The surface polariton propagation is restricted to be along the $x$-axis, parallel to the surface (Voigt geometry). The generalization for the QPS will be considered later.

The electric and magnetic fields are given by ( $s$ polarization):

$\vec{H}_{j}(x, y, z)=\left(H_{x j}, 0, H_{z j}\right) \exp \left(i k_{x} x-i \omega t\right)$,

$\vec{E}_{j}(x, y, z)=\left(0, E_{y j}, 0\right) \exp \left(i k_{x} x-i \omega t\right)$,

where for the magnetic layer we have

$$
\begin{aligned}
& H_{x A}(z)=A_{1 A}^{n} g_{A}+A_{2 A}^{n} \bar{g}_{A}, \\
& H_{z A}(z)=\left(-i / \mu_{0}\right)\left[\mu_{\mathrm{eff}}^{-} A_{1 A}^{n} g_{A}+\mu_{\mathrm{eff}}^{+} A_{2 A}^{n} \bar{g}_{A}\right], \\
& E_{y A}(z)=\left(i / k_{x}\right)(\omega / c)\left[\mu^{-} A_{1 A}^{n} g_{A}+\mu^{+} A_{2 A}^{n} \bar{g}_{A}\right],
\end{aligned}
$$

where $g_{A}=\exp \left(k_{A} z\right)$ and $\bar{g}_{A}=g_{A}^{-1}$. Also,

$\mu_{0}=-k_{x}^{2}+\mu_{1} \varepsilon_{A} \omega^{2} / c^{2}$,

$\mu_{\mathrm{eff}}^{ \pm}= \pm k_{x} k_{A}+\mu_{2} \varepsilon_{A} \omega^{2} / c^{2}$,

$\mu^{ \pm}=\mu_{2}-\mu_{1} \mu_{\mathrm{eff}}^{ \pm} / \mu_{0}$,

$k_{A}=\left[k_{x}^{2}-\mu_{V} \varepsilon_{A} \omega^{2} / c^{2}\right]^{1 / 2}$,

$\mu_{V}=\mu_{1}-\mu_{2}^{2} / \mu_{1}$,

with $\mu_{V}$ being the Voigt permeability. Here $k_{x}$ is the in-plane wave-vector, $\omega$ is the angular frequency, $c$ is the speed of light in vacuum, and $\varepsilon_{A}$ is the dielectric constant of medium $A$. One can show that for the metamagnetic material

$\mu_{1}=1+\frac{4 \pi\left(A_{1} \omega^{2}-B_{1}\right)}{\left(\omega^{2}-\omega_{1}\right)\left(\omega^{2}-\omega_{2}\right)}$,

$\mu_{2}=\frac{4 \pi\left(A_{2} \omega^{2}-B_{2}\right)}{\left(\omega^{2}-\omega_{1}\right)\left(\omega^{2}-\omega_{2}\right)}$,

with $A_{p}, B_{p}, \omega_{p}(p=1,2)$ defined elsewhere [10]. It is worthwhile to mention that $\omega_{1,2}$ represent the two resonant frequencies of the metamagnetic material. The first one, $\omega_{1}$, is related to the precession of the magnetization vector, $\vec{M}$, around the effective static field, defining the ferromagnetic phase. The second one, $\omega_{2}$, which is related to the precession of $\vec{M}$ around the exchange field $H_{E}$, defines the antiferromagnetic phase.

On the other hand, for the non-magnetic layer we have

$$
\begin{aligned}
& H_{x B}(z)=A_{1 B}^{n} g_{B}+A_{2 B}^{n} \bar{g}_{B}, \\
& H_{z B}(z)=\left(-i k_{x} / k_{B}\right)\left[A_{1 B}^{n} g_{B}-A_{2 B}^{n} \bar{g}_{B}\right], \\
& E_{y B}(z)=\left(i / k_{B}\right)(\omega / c)\left[A_{1 B}^{n} g_{B}-A_{2 B}^{n} \bar{g}_{A}\right],
\end{aligned}
$$

where $g_{B}=\exp \left(k_{B} z\right)$ and $\bar{g}_{B}=g_{B}^{-1}$. Also, $k_{B}=\left[k_{x}^{2}-\right.$ $\left.\varepsilon_{B} \omega^{2} / c^{2}\right]^{1 / 2}$, with $\varepsilon_{B}$ being the dielectric constant of medium $B$.

Defining, for each medium, the two column-vectors

$\left|A_{j}^{n}\right\rangle=\left[\begin{array}{l}A_{1 j}^{n} \\ A_{2 j}^{n}\end{array}\right]$,

and using Maxwell's boundary conditions on the interfaces $z=n L+d_{A}$ and $z=(n+1) L$, we find, in matrix form, the following equations for the amplitudes of the electromagnetic fields:

$$
\begin{aligned}
& M_{A}\left|A_{A}^{n}\right\rangle=N_{B}\left|A_{B}^{n}\right\rangle, \\
& M_{B}\left|A_{B}^{n}\right\rangle=N_{A}\left|A_{A}^{n+1}\right\rangle,
\end{aligned}
$$

where

$$
\begin{aligned}
M_{A} & =\left(\begin{array}{cc}
f_{A} & \bar{f}_{A} \\
\mu^{-} f_{A} & \mu^{+} \bar{f}_{A}
\end{array}\right), \\
M_{B} & =\left(\begin{array}{cc}
f_{B} & \bar{f}_{B} \\
-\left(k_{x} / k_{B}\right) f_{B} & \left(k_{x} / k_{B}\right) \bar{f}_{B}
\end{array}\right), \\
N_{A} & =\left(\begin{array}{cc}
1 & 1 \\
\mu^{-} & \mu^{+}
\end{array}\right), \quad N_{B}=\left(\begin{array}{cc}
1 & 1 \\
-k_{x} / k_{B} & k_{x} / k_{B}
\end{array}\right)
\end{aligned}
$$


with $f_{j}=\exp \left(-k_{j} d_{j}\right), \bar{f}_{j}=1 / f_{j}(j=A$ or $B)$. From Eqs. (17) and (18) it is easy to see that

$\left|A_{A}^{n+1}\right\rangle=T\left|A_{A}^{n}\right\rangle$,

where the $T$ matrix is given by $T=N_{A}^{-1} M_{B} N_{B}^{-1} M_{A}$. The $T$ matrix is called a transfer matrix because it relates the coefficients of the electromagnetic fields of one cell with those of the preceding cell. Taking into account the translational symmetry of our problem, we can use Bloch's theorem, to get the bulk polariton's dispersion relation in a periodic structure:

$\cos (Q L)=(1 / 2) \operatorname{Tr}(T)$.

Here $\operatorname{Tr}(T)$ means the trace of the matrix $T$, and $Q$ is the Bloch wave-vector.

To set up the dispersion relation of the surface polariton modes, we consider the periodic structure truncated at $z=0$ with the region $z>0$ filled by vacuum medium $C$, whose frequency-independent dielectric constant is denoted by $\epsilon_{C}$. This semi-infinite structure does not possess full translational symmetry in the $z$-direction through multiples of the unit-cell thickness $L$, and therefore we may no longer assume Bloch's ansatz as in the bulk case. However, Eq. (22) still holds provided we replace $Q$ by the complex quantity $i \beta$ to give $\cosh (\beta L)=$ $(1 / 2) \operatorname{Tr}(T)$. Since we now have to consider the extra boundary conditions for the new interface at $z=0$, this impose a further constraint which enables us eventually to determine the attenuation factor $\beta$. Applying the electromagnetic boundary conditions at $z=0$, the implicit dispersion relation for the surface polariton modes follows:

$T_{11}+\lambda T_{12}=\exp (-\beta L)=T_{21} \lambda^{-1}+T_{22}$,

where $\lambda=\left(k_{C}+k_{x} \mu^{-}\right) /\left(k_{C}-k_{x} \mu^{+}\right)$. Here $k_{C}$ is a pure real number, given by $k_{C}=\left[k_{x}-\omega^{2} / c^{2}\right]^{1 / 2}$. Also, the constant $\beta$ must be chosen to fulfill the requirement that $\operatorname{Re}(\beta)>0$, in order to give evanescent surface modes.

This algebraic method can be extended for quasiperiodic structures, by considering suitable transfer matrices $T$. Once these transfer matrices are determined, we must make use of Eqs. (22) and (23) to find the polariton's bulk and surface modes spectra, respectively.

Consider now the Fibonacci quasiperiodic structure. This structure can be grown experimentally by juxtaposing the two building blocks $A$ and $B$ in such a way that its $n$th generation $S_{n}$ is given iteratively by the rule $S_{n}=S_{n-1} S_{n-2}$, for $n \geqslant 2$, with $S_{0}=B$ and $S_{1}=A$. The infinite superlattice is also invariant under the transformation $A \rightarrow A B$ and $B \rightarrow A$. The number of blocks of the unit-cell grow with the Fibonacci number, $F_{l}=F_{l-1}+F_{l-2}\left(\right.$ with $\left.F_{0}=F_{1}=1\right)$, and the ratio between the number of building blocks $A$ and $B$ on the sequence is equal to the golden mean $\tau=(1 / 2)(1+\sqrt{5})$. The transfer matrix for the $n$th Fibonacci generations $(n \geqslant 1)$ is:

$T_{S_{n+2}}=T_{S_{n}} T_{S_{n+1}}$.

Therefore, with the knowledge of the above transfer matrix and the use of Eqs. (22) and (23), it is straightforward to get the polariton spectra discussed in the next section.

\section{Numerical results and conclusions}

In this section we will present our numerical results in order to characterize the spectra of the magnetic polaritons (bulk and surface modes), which can propagate on the Fibonacci quasiperiodic structure. To do that, we consider medium $A$ as the metamagnet $\mathrm{FeBr}_{2}$, in its ferromagnetic (FM) and antiferromagnetic (AFM) phases. The dielectric constant of this medium is $\varepsilon_{A}=1.5$. The approximate values of the exchange and anisotropy parameters are known from Raman scattering [11-13], and are given by: $J=5.07 \mathrm{~cm}^{-1}, H_{A}=7.34 \mathrm{~cm}^{-1}$, $\gamma M_{i}^{z}=1.2 \mathrm{kG}$ and $\gamma M_{j}^{z}=1.45 \mathrm{kG}$. For the $B$ medium, we consider the non-magnetic material $\mathrm{ZnF}_{2}$, whose dielectric constant is $\varepsilon_{B}=8$. We also define $R=d_{B} / d_{A}=0.5$ as the ratio between the thicknesses of the non-magnetic and magnetic layers, respectively. In the FM phase of the metamagnet $\mathrm{FeBr}_{2}$ we consider an applied field $\gamma H_{0}=20 \mathrm{kG}$, while for its AFM phase the magnetic field is set to zero.

The polariton spectra for the periodic and quasiperiodic (4thgeneration) Fibonacci structures are depicted in Figs. 1 and 2, respectively. The polariton spectra are graphically represented as the reduced frequencies $\omega / \omega_{\mathrm{FM}}$, for the metamagnet $\mathrm{FeBr}_{2}$ in its $\mathrm{FM}$ phase, and $\omega / \omega_{\mathrm{AFM}}$, for the metamagnet $\mathrm{FeBr}_{2}$ in its AFM phase, versus the dimensionless wave-vector $k_{x} d_{A}$. In all figures the surface modes are represented by solid lines, while the bulk modes are represented by the shaded regions limited by the curves $Q L=0$ and $Q L=\pi$. The dashed lines, from which the surface modes arise, represent the light line in vacuum.

For the periodic case (which is equivalent to the second Fibonacci generation), Fig. 1(a) shows, for the metamagnet $\mathrm{FeBr}_{2}$ in its FM phase, two well defined bulk branches: a high- frequency region, in the range $1.3<\omega / \omega_{\mathrm{FM}}<2.8$, and a lowfrequency one, limited by the range $0<\omega / \omega_{\mathrm{FM}}<1.2$. Considering the AFM phase (Fig. 1(b)), the bulk bands profile have a similar behavior, with a high-frequency region limited by $2.25<\omega / \omega_{\mathrm{AFM}}<4.6$, and a low-frequency one, defined by $0<\omega / \omega_{\text {AFM }}<1.7$. The surface modes (solid lines) always display a non-reciprocal behavior, namely $\omega(k) \neq \omega(-k)$ [14].

In Fig. 2 we can see a more complex magnetic polariton spectra for the fourth Fibonacci generation. One can notice that the number of bulk bands is bigger then in the periodic case, being equal to the Fibonacci number $F_{4}=5$, and indeed, usually this number is always equal to the Fibonacci number for the corresponding generation. This feature was observed for other excitations [15], although with other relation between the number of bands and the generation considered, and can be physically interpreted as follow: when the quasiperiodicity is introduced, the complex arrangement of magnetic and non-magnetic layers imposes constraints on the possible frequencies (or energies), acting in a similar way as the constraints on the Lagrange formalism describing the motion of a mechanical system (the Lagrangian is a function of the energies of a mechanical system). For a given wave-vector, the propagation of the magnetic polaritons is then subject to these constraints. This in turn gives rise to the richer allowed and forbidden bands scheme, as can be seen on Figs. 2(a) and 2(b), and with the complexity of the spectrum increasing as one goes to higher generations of the 

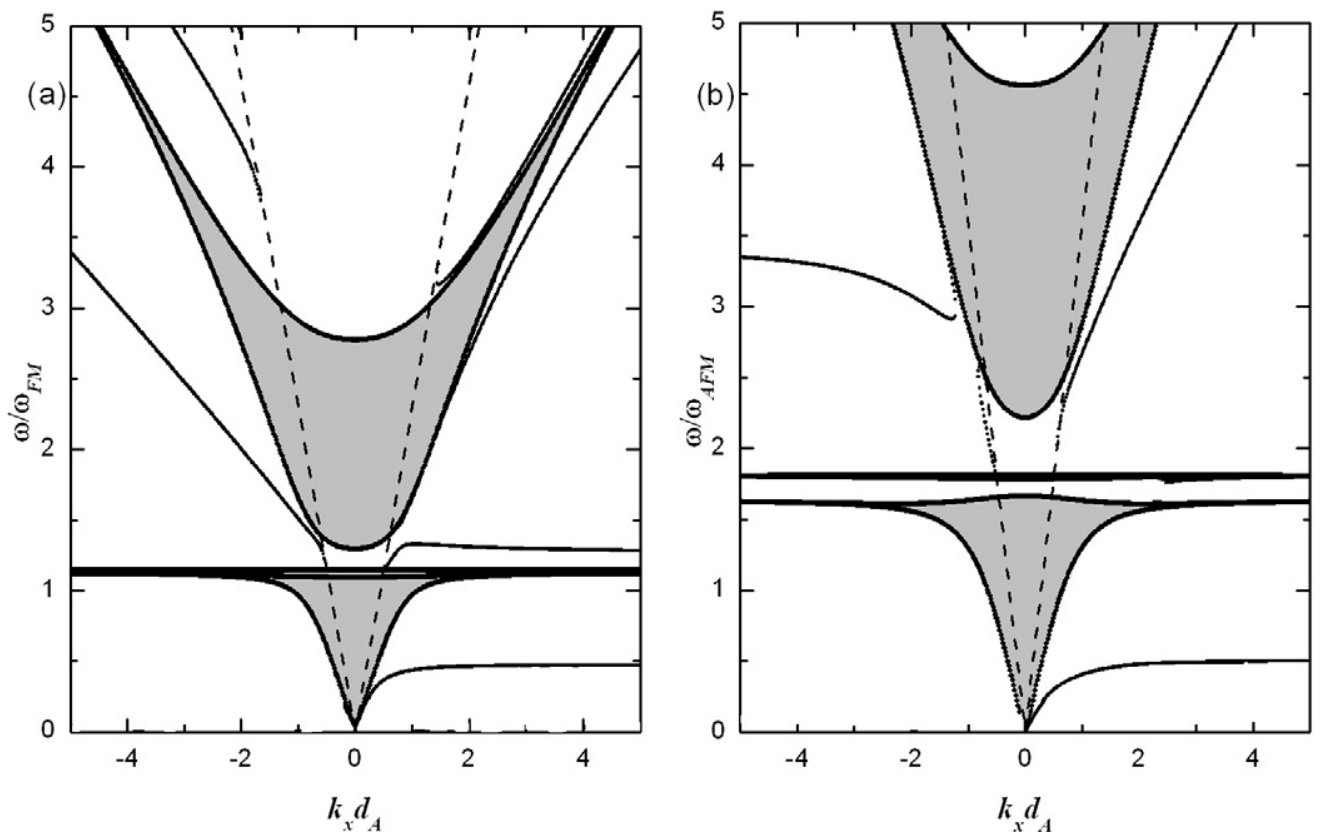

Fig. 1. (a) Magnetic polariton spectra given by the reduced frequency $\omega / \omega_{\mathrm{FM}}$ as a function of the dimensionless wave-vector $k_{x} d_{A}$ for the metamagnet $\mathrm{FeBr}_{2}$ in its FM phase; (b) same as in (a), but considering the reduced frequency $\omega / \omega_{\mathrm{AFM}}$ for the metamagnet $\mathrm{FeBr}_{2}$ in its AFM phase.
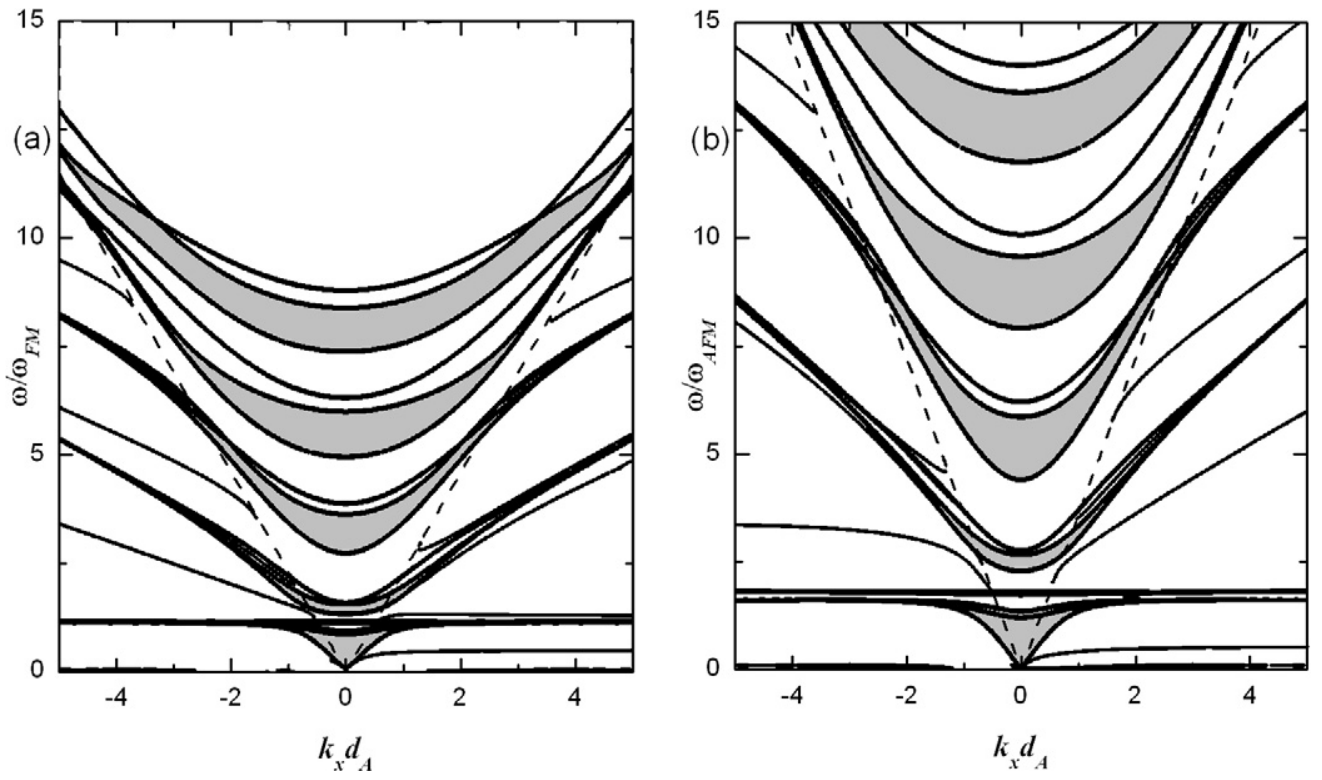

Fig. 2. Same as in Fig. 1 but for the fourth Fibonacci generation.

Fibonacci unit-cell. The surface modes exhibit a non-reciprocal behavior, as in the periodic case.

In order to characterize better the fractal aspect of the magnetic polariton spectra, we now examine the confinement effects arising from competition between the long-range aperiodic order, induced by the quasiperiodic structure, and the short-range disorder. To this end, a quantitative analysis should be made of the localization and magnitude of the allowed bandwidth in the polariton spectra. We investigate also their scaling behavior, as a function of the sequence's generation number.

The bandwidth distribution is shown on Fig. 3, for the dimensionless wave-vector $k_{x} d_{A}=2.5$. It can be seen the allowed and forbidden frequency regions for the bulk polariton propagation, as a function of the generation number $n$, up to the tenth generation of the Fibonacci sequence (Fig. 3(a)), which means an unit-cell with 55 blocks of material $A$ (metamagnetic) and 34 blocks of material $B$ (non-magnetic). Fig. 3(b) depicts the allowed regions, up to the 12 th Fibonacci generation, with an unit-cell made up by $144 A$ blocks and $89 B$ blocks. It can be seen clearly the localization of the modes, when it goes to higher values of the sequence generation (for higher values of $n$, the allowed bands get narrow and narrower, forming a typical Cantor set structure). The self-similar feature of the spectrum is shown on the rectangular region on Fig. 3(a), which is repro- 

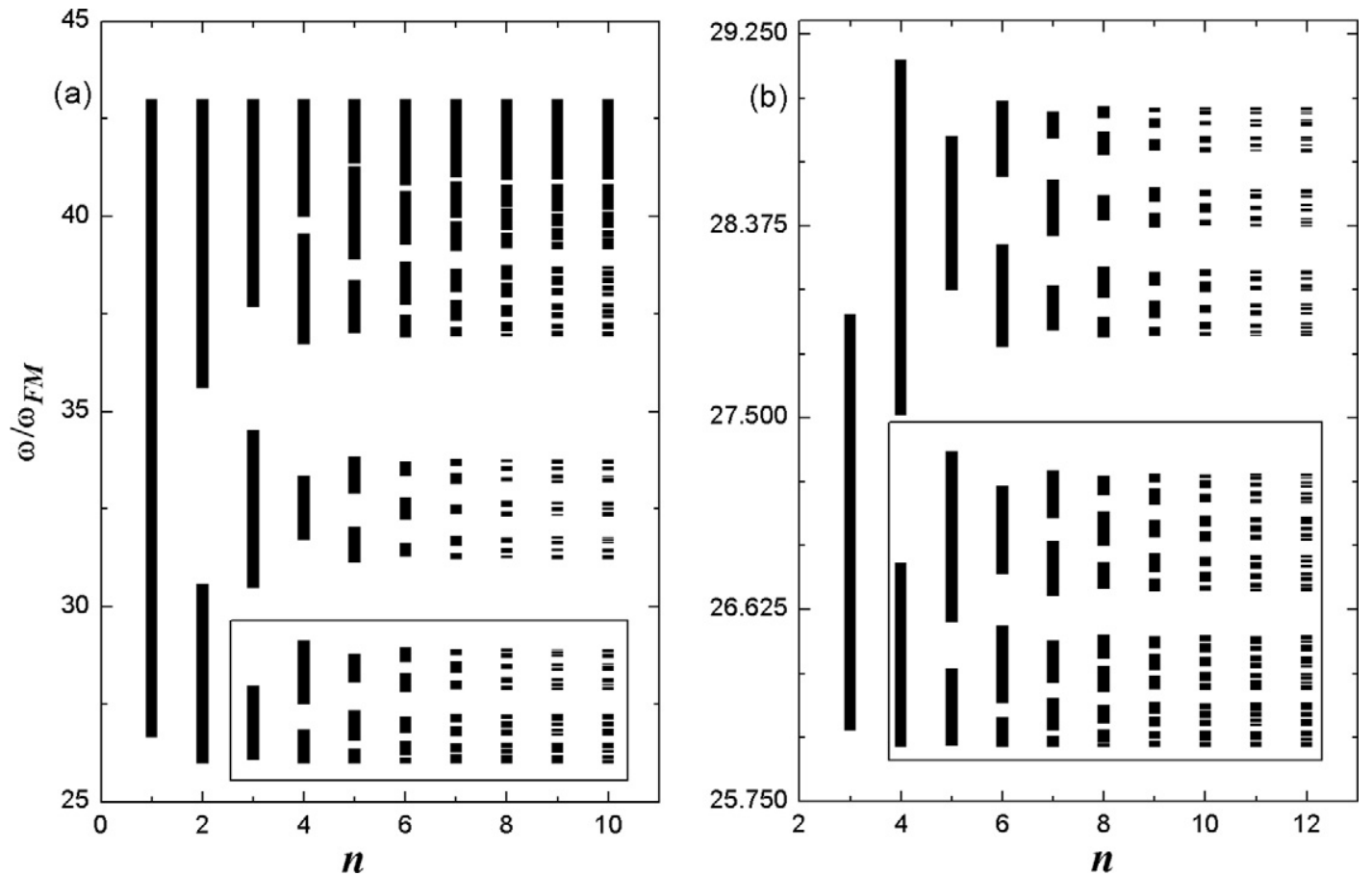

Fig. 3. (a) Bandwidth distribution of the magnetic polaritons for $k_{x} d_{A}=2.5$. The rectangle shows the window where the spectra is self-similar. (b) Same as in (a), but for an enlarged region. The new rectangle characterizes another window where the spectrum is self-similar.

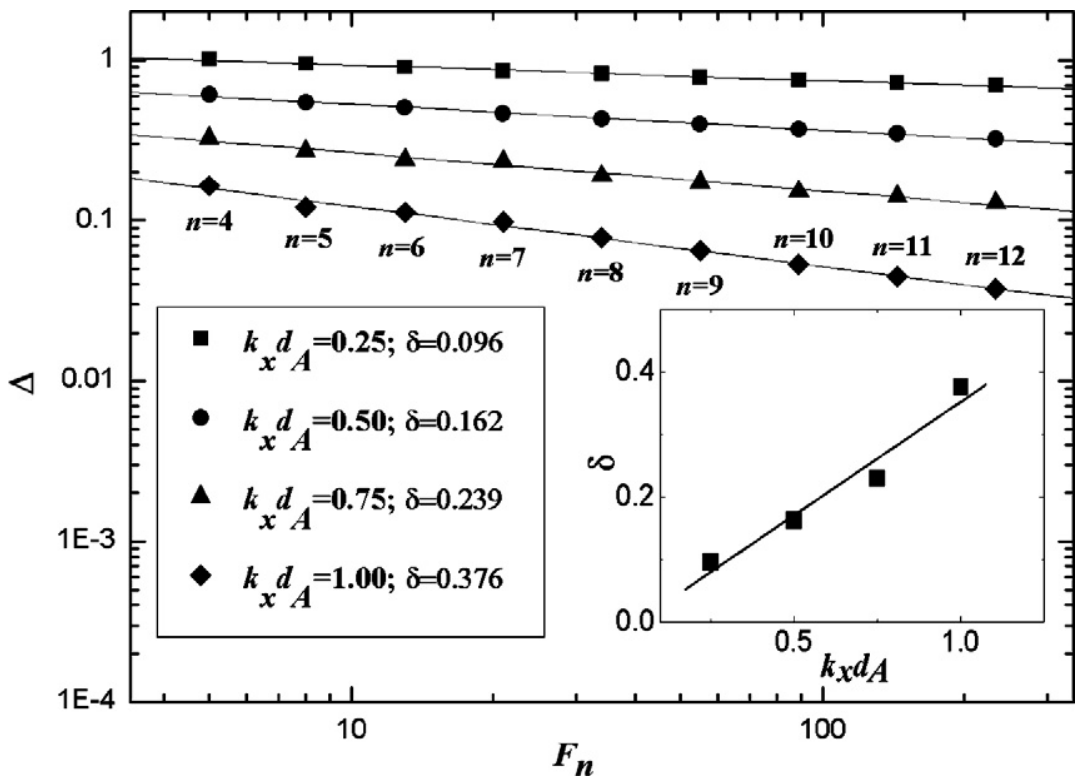

Fig. 4. Log- $\log$ representation of the total width of the allowed regions $\Delta$ versus the Fibonacci number $F_{n}$.

duced, in a small scale, on Fig. 3(b). Actually, this property of self-similarity is a typical feature of a fractal set, and is a consequence of the coupling between the set up method employed to obtain the superlattice, together with the properties of the excitation considered, namely, the magnetic polaritons. A similar property was found in previous works [16] considering the spin waves spectra of a quasiperiodic Fibonacci magnetic structure, with the metamagnet $\mathrm{FeBr}_{2}$ as the magnetic material, and for magnetostatic modes in antiferromagnetic materials [15].
The total summation of the widths of these regions (or energy bands), represented by $\Delta$, scales with the Fibonacci number as a power law $\Delta \sim F_{n}^{-\delta}$, where $F_{n}$ is the $n$th Fibonacci number and $\delta$ is a scaling index, which is a function of $k_{x} d_{A}$, and can be interpreted as a diffusion coefficient. In Fig. 4 we show a $\log -\log$ plot, of these power laws for four different values of $k_{x} d_{A}$ (namely, $0.25,0.5,0.75,1.0$ ). Observe in the inset of this figure the linear, monotonically increasing, behavior of the scale exponent $\delta$ as a function of the in-plane common 
wave-vector $k_{x} d_{A}$. The good linear fit for the Fibonacci case is probably due to its relatively small growth rate, and consequent smaller localization.

In summary, this work deals with a general theory for the propagation of magnetic polaritons on quasiperiodic structures of Fibonacci type, made up by the stacking of two different layers, where one of them is a metamagnetic thin film $A$, while the other is a non-magnetic insulator thin layer $B$. Some important physical properties of these structures are also investigated, mainly those related to their selfsimilar behaviors, whose fractal aspect can be described in terms of power laws, as shown in Fig. 4, including the bandwidth distribution of their spectra, depicted in Figs. 3(a) and 3(b).

\section{Acknowledgements}

We acknowledge the financial support received from the Brazilian National Research Council $(\mathrm{CNPq})$, projects CTENERG 554889/2006-4 and CNPq-Rede NanoBioestruturas $555183 / 2005-0$.

\section{References}

[1] D. Shechtman, I. Blench, D. Gratias, J.W. Cahn, Phys. Rev. Lett. 53 (1984) 1951.

[2] E.L. Albuquerque, M.G. Cottam, Polaritons in Periodic and Quasiperiodic Structures, Elsevier, Amsterdam, 2004.

[3] E.L. Albuquerque, M.G. Cottam, Phys. Rep. 376 (2003) 225.

[4] F. Hippert, D. Gratias (Eds.), Lectures on Quasicrystals, Les Editions de Physique, Les Ulis, 1994.

[5] P. Dhez, C. Weisbuch (Eds.), Physics, Fabrication and Applications of Multilayered Structures, Plenum, New York, 1988

[6] J.H. Baskey, M.G. Cottam, Phys. Rev. B 42 (1990) 4304.

[7] D.H.A.L. Anselmo, E.L. Albuquerque, M.G. Cottam, J. Appl. Phys. 83 (1998) 6955.

[8] E. Meloche, M.G. Cottam, Phys. Rev. B 70 (2004) 094423.

[9] J.M. Pereira, M.G. Cottam, Phys. Rev. B 63 (2001) 174431.

[10] E.S. Guimaraes, E.L. Albuquerque, Phys. Lett. A 307 (2003) 172.

[11] W.B. Yelon, C. Vettier, J. Phys. C 8 (1975) 2760.

[12] G.C. Psaltakis, G. Mischler, D.J. Lockwood, M.G. Cottam, A. Zwick, S. Legrand, J. Phys. C 17 (1984) 1735.

[13] G.C. Psaltakis, M.G. Cottam, J. Phys. C 15 (1982) 4847.

[14] R.E. Camley, Surf. Sci. Rep. 7 (1987) 103.

[15] M.S. Vasconcelos, D.H.A.L. Anselmo, C.G. Bezerra, Solid State Commun. 135 (2005) 673.

[16] M.S. Vasconcelos, P.W. Mauriz, U.L. Fulco, E.L. Albuquerque, Phys. Lett. A 363 (2007) 322 . 